\title{
Clinical, Pathological, and Prognostic Analysis of Urachal Carcinoma
}

\author{
Guangjun Shao ${ }^{a}$ Chunru Xub,c,d Jikai Liu ${ }^{a}$ Xuesong Li ${ }^{b, c, d}$ Luchao $\mathrm{Li}^{\mathrm{a}}$ \\ Xiaofeng $\mathrm{Li}^{\mathrm{a}}$ Xiaoqing Zhang ${ }^{\mathrm{b}, \mathrm{c}, \mathrm{d}}$ Yidong Fan ${ }^{\mathrm{a}}$ Liqun Zhou ${ }^{\mathrm{b}, \mathrm{c}, \mathrm{d}}$ \\ aDepartment of Urology, Qilu Hospital, Cheeloo College of Medicine, Shandong University, Jinan, China; \\ bepartment of Urology, Peking University First Hospital, Beijing, China; Institute of Urology, Peking University, \\ Beijing, China; ${ }^{d}$ National Urological Cancer Center, Beijing, China
}

\section{Keywords}

Urachal carcinoma - Extended partial cystectomy ·

Prognosis · High-risk TNM stage · Positive surgical margin ·

Adjuvant therapy

\begin{abstract}
Objective: The aim of this study was to improve understanding the clinical, pathologic, and prognostic features of urachal carcinoma (UrC), a retrospectively descriptive study was done in 2 clinical centers. Methods: After excluding the 2 missed patients, the clinical and pathological data of 59 patients with UrC, who were diagnosed or treated at 2 clinical centers between 1986 and 2019, was retrospectively analyzed. SPSS 22.0 (IBM) and GraphPad Prism 8.0.1 were used for statistics and data visualization. Survival data were analyzed by the Kaplan-Meier method and Log-rank tests. Cox proportional hazards regression were performed for find risk factors on predicting the prognosis. Results: Of all 59 patients, 47 were male and 12 were female. The median age at diagnosis was 51.6 years (range: 22-84 years). Gross hematuria was the most common symptom (79.66\%). The majority of urachal neoplasms were adenocarcinomas (94.92\%). Forty-two patients (72.41\%) underwent extended partial cystectomy with en bloc resection of the entire urachus. The
\end{abstract}

mean follow-up was 52 months (3-277 months). Median overall survival was 52.8 months (4-93 months). The 3-year cancer-specific survival (CSS) rate and 5-year CSS rate were $69.1 \%$ and $61.2 \%$. There was no significant difference among localized T stage, tumor histologic grade and surgical procedures in determining prognosis by survival analyze. While patients with high-risk TNM stage (local abdominal metastasis, lymph node metastasis, or distant metastasis) ( $p=0.003$ ) and positive surgical margin $(p<0.001)$ had significantly worse prognosis. Conclusions: The results indicate that high-risk TNM stage and positive surgical margin are risk predictors of prognosis. Localized T stage, histologic grade, and surgical procedure cause no significant effect on patient prognosis. The extended partial cystectomy is the recommended surgical approach for patients with UrC. Active multimodal treatments may improve the survival of patients with recurrent and metastatic disease.

(c) 2021 The Author(s).

Published by S. Karger AG, Basel

Guangjun Shao works now at the Department of Urology, the People's Hospital of Fushan District, Yantai, Shandong 265500, China; Email: shgj2004@163.com.

G.S. and C.X. contributed equally to this work and should be considered co-first authors. karger@karger.com www.karger.com/uin

Karger $\stackrel{\text { ' }}{5}$
(C) 2021 The Author(s)

Published by S. Karger AG, Basel

This is an Open Access article licensed under the Creative Commons Attribution-NonCommercial-4.0 International License (CC BY-NC) (http://www.karger.com/Services/OpenAccessLicense), applicable to the online version of the article only. Usage and distribution for commercial purposes requires written permission.
Correspondence to:

Yidong Fan, fanyd@ sdu.edu.cn

Liqun Zhou, zhoulqmail@ sina.com 


\section{Introduction}

The urachus is a thick fibrous cord and is located preperitoneal in a pyramid-shaped space. It is a tubular channel that connects the fetal bladder to the urinary bladder during fetal life and progressively occludes the official cavity as it develops, leaving only striated structures connecting the anterior dome of the bladder with the umbilicus [1]. From outside to inside, it consists of 3 layers of the smooth muscle, submucosal connective tissues, and urothelial cells tissues. Urachal neoplasms can be found in any of these segments $[1,2]$.

Urachal carcinoma ( $\mathrm{UrC}$ ) is a rare malignancy accounting for only $0.35-0.7 \%$ of all bladder cancers [3]. Patients with UrC are usually diagnosed with local progression or extensive metastases at their first visit, which means a poor prognosis. However, some studies indicated a rather higher cancer-specific survival (CSS) rate of UrC than nonurachal adenocarcinoma and urothelial carcinoma of the bladder $[4,5]$.

To the best of our knowledge, previous studies about $\mathrm{UrC}$ are sparse, and the optimal treatment is still a matter of debate [6]. In this study, we retrospectively reviewed our experiences of treatment with $\mathrm{UrC}$ to investigate the clinical, pathologic, and prognostic features of this rare neoplasm.

\section{Materials and Methods}

\section{Patients Selection}

This retrospective multicenter study was approved by the institutional review boards. After excluding 2 missing cases, records of 59 cases diagnosed and treated as $\mathrm{UrC}$ at our institution between January 1986 and December 2019 were included. Among these patients, except one had developed distant metastasis, all underwent surgical intervention and pathology diagnosis. Patients' demographics, clinical features, treatments, pathological and clinical outcomes were studied in this analysis. Patients' treatment information was obtained through the clinical His system. Two investigators (G.J.S. and X.Q.Z.) were responsible for following up with patients by phone or mail. The accuracy of the final information was checked and confirmed by a third investigator (C.R.X.). All enrolled patients completed an informed consent form before receiving treatment.

\section{Definition of Variables for Analyses}

Variables included in this study included patients' gender, age, smoking history, time of diagnosis, Imaging data, TNM stage, pathological grade, surgical procedure, type of postoperative pathology, presence of lymph node metastases, abdominal metastases, distant metastases, and positive cut margins. The overall survival (OS), recurrence-free survival (RFS), and CSS were the main goal outcome in our analysis.

\section{Pathological Diagnostic Criteria}

The criteria for the diagnosis of UrC according to the modified Sheldon criteria are as follows [2]: (a) tumor was located in the dome or anterior wall of the bladder; (b) tumor extended into the bladder wall; (c) absence of cystitis cystic or glandular in the dome or anterior wall of the bladder; (d) absence of a known primary cancer elsewhere. The TNM staging system was universally applied and used to determine prognosis in the current study [7] (the TNM staging system for urachal cancer is shown in Table 1).

\section{Statistical Analyses}

Survival data were analyzed by the Kaplan-Meier method. For estimation of RFS, CSS, and OS, Kaplan-Meier curves were created; the comparison was done with the log-rank test. Univariable and multivariable Cox regression analyses were performed to evaluate the prognostic effect of $\mathrm{UrC}$ on survival. Predictive value on definitive pathologic features was measured with univariable and multivariable logistic regression analyses. Statistical analyses were performed using IBM SPSS 22.0 (IBM, Chicago, IL, USA). All tests were 2 -sided, and $p<0.05$ was considered statistically significant. GraphPad 8.0.1 (GraphPad, San Diego, CA, USA) was used for data visualization.

\section{Results}

\section{Patient Demographics and Clinical Features}

Of the 59 patients with $\mathrm{UrC}$, there were 47 males and 12 females (male to female ratio 3.9:1). The median age at diagnosis was 51.6 years (range: $22-84$ years). Patients with a smoking history were in 19 cases (32.2\%).

The most common symptom was gross hematuria (79.66\%); other symptoms included lower urinary tract symptoms (15.25\%), abdominal mass (8.47\%), mucinuria (6.78\%), and abdominal pain (11.86\%). The urachal tumors were incidentally found on a radiographic checkup for other causes in 6 cases $(10.17 \%)$.

Abdominal ultrasonography, computed tomography (CT), and cystoscopy would be the main tests for diagnosing urachal tumors. Fifty-one patients underwent abdominal ultrasonography and the most common finding was a mass observed at the dome or frontier wall of the bladder $(98.08 \%)$. CT scans were performed in 44 patients. Of these patients, there were 41 cases $(93.18 \%)$ with a solid mass and 3 cases $(6.82 \%)$ with a cystic mass. Calcification was observed in 10 cases $(22.73 \%)$ (shown in Fig. $1 \mathrm{a}-\mathrm{c}$ ). A total of 46 patients underwent preoperative cystoscopy or biopsy, with the most common intraoperative description being a predominant manifestation visible in the top midline or the anterior wall of the bladder. Malignant tumors were found at the dome of the bladder by magnetic resonance imaging in 4 cases (shown in Table 2). 



Fig. 1. a-c CT images show a solid mass in the dome of the bladder with calcification. CT, computed tomography.

Fig. 2. The resected specimen of umbilical, urachus, and urachal carcinoma.

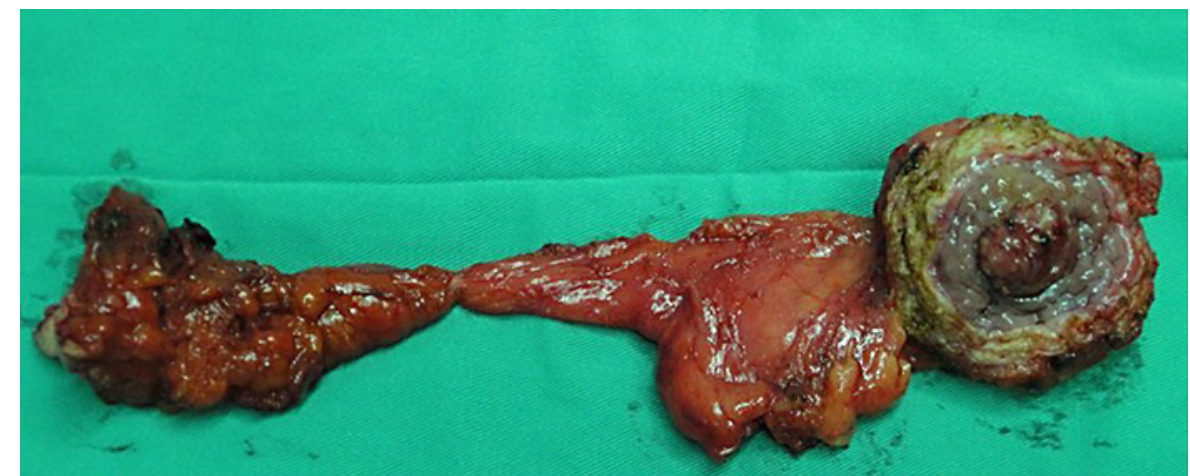

Table 1. The TNM staging system for urachal cancer

\begin{tabular}{ll} 
The TNM staging system & \\
\hline TNM & Description \\
Tis & A tumor localized to the urachal mucosa and no invasion to the basal membrane (carcinoma in situ) \\
T1 & A tumor with invasion through the basal membrane \\
T2 & Tumor invades into muscle of the bladder \\
pT2a & Tumor invades deep muscle of the bladder (outer half) \\
pT2b & Tumor invades the superficial muscle of the bladder (inner half) \\
T3 & Tumor invades perivesical fat, abdominal wall muscle (in cases of extravesical urachal cancers) \\
N & Followed the traditional TNM staging system \\
M & Peritoneal implants were considered metastasis
\end{tabular}

\section{Pathologic Outcomes}

The average size of urachal tumors was $3.9 \mathrm{~cm}$ (range: $1.3-8.0 \mathrm{~cm}$, the resected specimen shown in Fig. 2). Of 59 urachal cancers, the tumor was located in the dome of the bladder in 47 cases $(76.09 \%)$ and at the anterior wall of the bladder in 10 cases $(21.74 \%$ ) ( 2 cases were multiple tumors). Histologically, 19 cases were adenocarcinoma not otherwise specified (NOS), 19 cases were mucinous adenocarcinoma, 5 cases were adenocarcinoma with mu-

The Characteristics of Urachal Carcinoma cinous adenocarcinoma, 5 cases were mucinous adenocarcinoma with signet ring cells, and 2 cases showed adenocarcinoma mixed with signet ring cells and mucous cells or enteric differentiation. Only 1 case showed pure signet ring cell subtype, and 5 cases exhibited enteric adenocarcinoma. Two cases were small-cell carcinoma and 1 case was transitional-cell carcinoma with adenomatous metaplasia (shown in Fig. 3a-c). Lymph node invasion was present in 6 patients. The positive surgical margin, 
Table 2. Patient demographics and clinical features of 59 cases with UrC

\begin{tabular}{lrcc}
\hline & Cases, $N$ & Percent, $\%$ & $p$ value \\
\hline Gender & & & \\
$\quad$ Male & 47 & 79.66 & $<0.001$ \\
$\quad$ Female & 12 & 20.34 & \\
Smoking history & & & \\
$\quad$ Yes & 19 & 32.20 & $<0.001$ \\
$\quad$ No & 40 & 67.80 & \\
Symptom & & & \\
$\quad$ Gross hematuria & 47 & 79.66 & \\
LUTS & 9 & 15.25 & \\
Abdominal mass & 5 & 8.47 & \\
$\quad$ Mucinuria & 4 & 6.78 & \\
$\quad$ Abdominal pain & 7 & 11.86 & \\
$\quad$ Other & 6 & 10.17 & \\
USA (find mass) & & & \\
$\quad$ Yes & 51 & 98.08 & $<0.001$ \\
$\quad$ No & 1 & 1.92 & \\
CT & & & \\
Solid mass & 41 & 93.18 & \\
Cystic mass & 3 & 6.82 & $<0.001$ \\
$\quad$ Calcification & 10 & 22.73 & \\
MRI & & & \\
$\quad$ Malignant tumor & 4 & 100.00 & \\
Cystoscopy & & & \\
$\quad$ Mass at dome & 35 & 76.09 & \\
$\quad$ Mass at anterior wall & 10 & 21.74 & \\
$\quad$ Multiple & 1 & 2.17 & \\
\hline
\end{tabular}

UrC, urachal carcinoma; LUTS, lower urinary tract symptoms; CT, computed tomography; MRI, magnetic resonance imaging.

defined as tumor cells were seen microscopically at any part of the proximal cut edge of umbilical ureteral or resection margin of bladder, was seen in 2 cases.

According to the TNM staging system for urachal cancer, Tis tumors were not diagnosed in any cases and the $\mathrm{T} 1$ stage was seen in one case. The tumors were T2 in 17 cases, T3 in 38 cases (64.41\%), and stage IV (TxN1-2/M1 and local abdominal metastasis) in 13 cases (shown in Table 3).

\section{Localized Treatment}

Except one had developed distant metastasis, all patients were treated with surgical interventions. Extended partial cystectomy, including resection with or without the umbilicus, was the most used surgical procedure in 42 patients $(72.41 \%)$. The anatomic boundaries of resection included resection of the tumor, with at least $2-3 \mathrm{~cm}$ macroscopically normal bladder margins; the entire length of the umbilical ureter, the transverse abdominal fascia, and part of the peritoneum. The entire top of the bladder was removed for tumors located on the dome, and the peritoneum of the top of the bladder was separated in advance then the abdominal cavity was closed. Ten patients underwent partial cystectomy, and 6 patients underwent radical cystectomy. Bilateral lymphadenectomy, containing at least obturator, internal iliac, and external iliac lymph node stations as well as visible hyperplasia, was performed in 21 of all patients. All specimens were routinely processed for histologic examination.

\section{Adjuvant Treatment and Treatment for Recurrencel Metastasis}

Adjuvant chemotherapy was performed in 11 patients for locally advanced disease or distant metastasis and in 8 patients for postoperative local recurrence or distant metastasis. Several chemotherapy regimens were used in the past 30 years. The main aspect of the regimens was based on the combination of 5-fluorouracil (5-FU) or gemcitabine and/or cisplatin. Radiation therapy was given as adjuvant treatment in 4 cases for control of metastatic. In sum, 2 patients were treated with concurrent adjuvant chemotherapy and radiotherapy.

\section{Clinical Follow-Up and Predictors of Prognosis}

Fifty-nine patients were followed up, with a mean follow-up of 52 months (range: 3-277 months). Twenty-two patients died of $\mathrm{UrC}$ in a mean of 52.8 months (range: 4-93 months). The median OS of UrC patients was 52.8 months and median RFS was 20 months (shown in Fig. 4). Cox regression was done to find 2 factors as high-risk TNM stage and positive surgical margin affected prognosis significantly. Kaplan-Meier survival curve revealed the overall 3-year and 5-year CSS rates were $69.1 \%$ and $61.2 \%$, respectively. A log-rank test revealed that the CSS rate was significantly different with a positive surgical margin $(p<$ 0.001 ) and with or without local progression (lymph node or abdominal metastasis) and distant metastasis ( $p=$ 0.003 ). While the RFS was only associated with the positive surgical margin $(p=0.019)$. It was not significantly different in either CSS and RFS of patients with the factors like the localized T stage, tumor histologic grade $(G)$, and surgical procedure in the Log-rank test (shown in Fig. 5).

\section{Discussion}

$\mathrm{UrC}$ is an extremely rare urologic tumor, which was first described by Begg [8], originated from the remnant of the urachus. It is always located in the dome or ante- 
Table 3. Pathological features and surgical treatments of $\mathrm{UrC}$

Cases, $N \quad$ Percent, $\% \quad p$ value

Histology

TNM stage

Grade

Adenocarcinoma NOS

Mucinous adenocarcinoma

Adenocarcinoma + mucinous adenocarcinoma

Mucinous adenocarcinoma + signet ring cell

Mixed adenocarcinoma

Enteric adenocarcinoma

Signet ring-cell carcinoma

Small-cell carcinoma

Transitional-cell carcinoma with adenomatous metaplasia

Tis

T1

$\mathrm{T} 2$

T3

IV

Well differentiated

Moderately differentiated

Poorly differentiated

Surgical approach

Extended partial cystectomy

Partial cystectomy

Radical cystectomy

Lymph node dissection

Positive

Positive surgical margin

Local abdominal metastasis

Adjuvant chemotherapy

Adjuvant radiotherapy

19

19

5

5

2

5

1

UrC, urachal carcinoma; NOS, not otherwise specified.
32.20

32.20

8.47

8.47

3.39

8.47

1.69

3.39

1.69

0

1.69

28.81

64.41

22.03

12.07

53.45

34.48

72.41

17.24

10.34

28.57

3.39

18.64

30.51

6.78
$<0.001$

$<0.001$

$<0.001$

$<0.01$


Fig. 3. a Urachal adenocarcinoma, mucinous type. b Urachal adenocarcinoma, signet ring cell type. c Urachal small cell carcinoma (HE.; original magnification $\times 200)$. 
rior wall of the bladder. The majority of histological types are adenocarcinomas, included mucinous, enteric, mixed, and signet ring cell types $[2,3,9]$. Urachal adenocarcinoma accounts for approximately only $10 \%$ of primary bladder adenocarcinoma [4]. Other rarer urachal neoplasms include urothelial carcinoma, squamous cell carcinoma, small cell carcinoma, and sarcomas $[7,10]$. Although there is still controversy over the origin of $\mathrm{UrC}$, the glandular metaplasia basis and the origin of glandular intestinal epithelial cells are the most commonly accepted theories to explain the predominance of adenocarcinoma of the UrC [5].

$\mathrm{UrC}$ usually occurs in the $50 \mathrm{~s}$ and $60 \mathrm{~s}$ and is more common in men according to previous reports $[3,5]$. This is consistent with our observations (the median age of patients was 51.6 years, and the male to female ratio was 3.9:1). In the current retrospective study, the most common presenting symptom was gross hematuria, which was in agreement with the previous reports [11].

Until now, the standard diagnostic criteria of UrC was still in debate. Several staging systems had been proposed for UrCs, such as the Sheldon et al. [12], the Mayo [11], and the Ontario [13] staging systems. Most scientists and clinicians agreed with the criteria proposed by Sheldon and revised by Gopalan et al. [2]. Ultrasonography most frequently shows a supra-vesical, inhomogeneous, and irregular mass in the midline [14]. CT findings were also very useful in TNM staging and diagnosing urachal tumors especially midline masses with calcification. However, only half of the bladder wall invasions can be detected by CT, showing its limited value in estimating tumor invasion [15]. Since the UrC and urothelial carcinoma were often difficult to differentiate by imaging alone, cystoscopy and pathological biopsy were extremely important to confirm the diagnosis [11].

Surgical approaches still played a role as the primary treatment for patients diagnosed with $\mathrm{UrC}$ without distant metastasis [16]. Furthermore, extended partial cystectomy with en bloc resection of the urachal tumor, the entire urachus, and umbilicus, and pelvic lymph node dissection was the recommended surgery approach applied by most urologists $[13,17,18]$. There is still controversy as to which is better between partial cystectomy and expanded partial bladder resection. And the existing data demonstrated that radical cystectomy was not superior to extended partial cystectomy, and radical cystectomy did not improve survival compared to partial cystectomy [13, 18]. Laparoscopic extended partial cystectomy had been

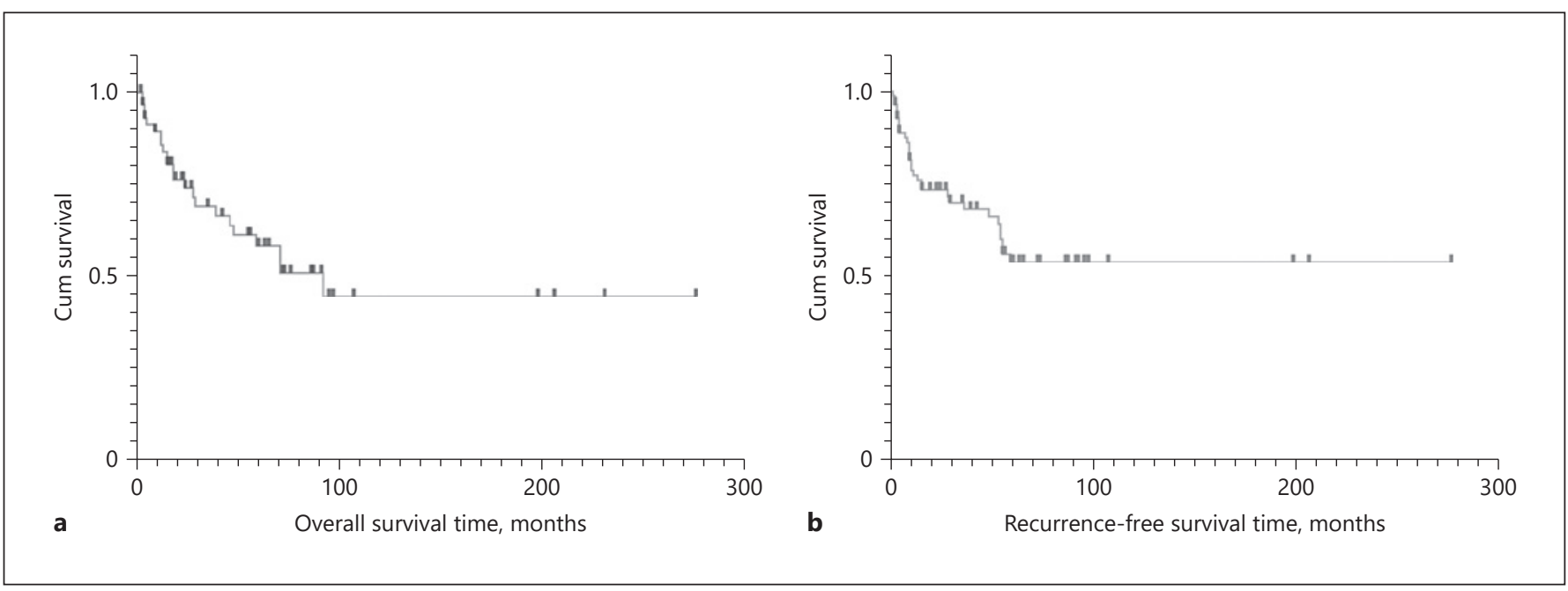

Fig. 4. Overall survival (a) and recurrence-free survival (b) of patients with urachal carcinoma.

Fig. 5. Kaplan-Meier survival curve of different risk factors of CSS and RFS: a1, a 2 by localized T stage (CSS $p=0.588$ RFS $p=0.748$ ); b1, b2 by histologic grade (CSS $p=0.12$; RFS $p=0.19$ ); $\mathbf{c 1 , ~ c 2 ~ b y ~}$ surgical procedure (CSS $p=0.738$; RFS $p=0.677$ ); $\mathbf{d} 1$, $\mathbf{d} 2$ by local progression and distant metastasis (CSS $p=0.003$; RFS $p=0.11$ ); e1, e2 by positive surgical margin (CSS $p<0.001$; RFS $p<0.001$ ). AUS, abdominal ultrasonography; CSS, cancer-specific survival; LN, lymph node; NOS, adenocarcinoma not otherwise specified; OS, overall survival; RFS, recurrence-free survival.

(For figure see next page.) 




a1 Cancer-specific survival time, months

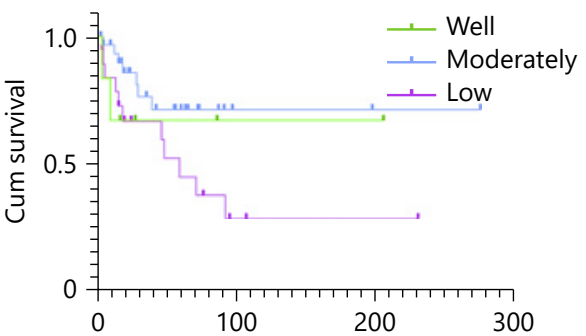

b1 Cancer-specific survival time, months

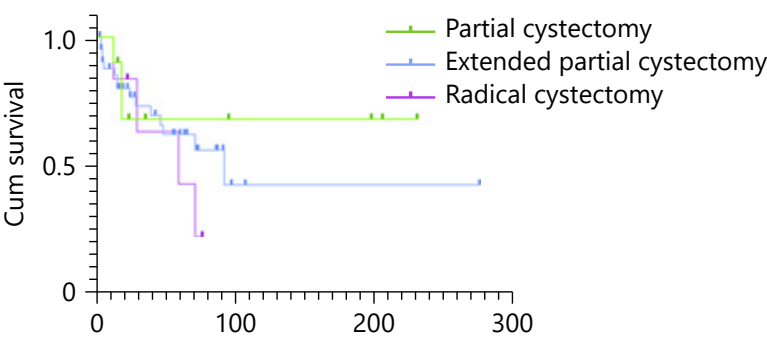

c1 Cancer-specific survival time, months

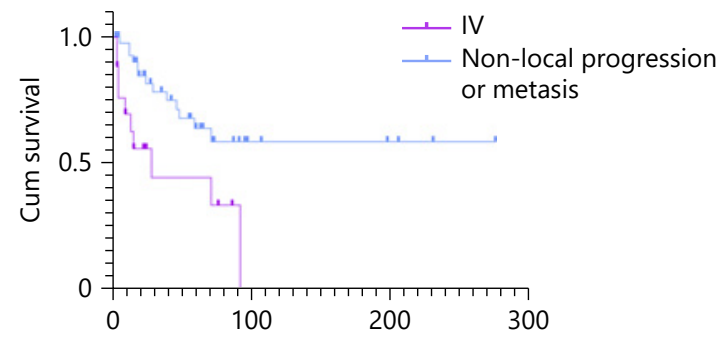

d1 Cancer-specific survival time, months

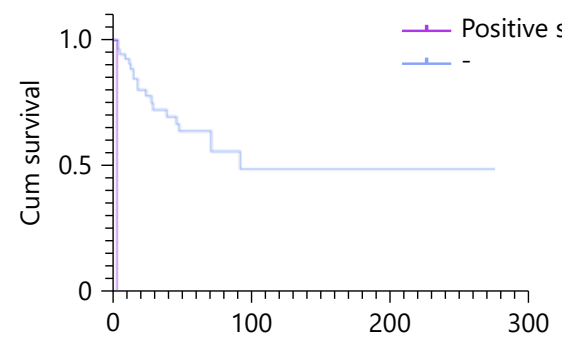

e1 Cancer-specific survival time, months

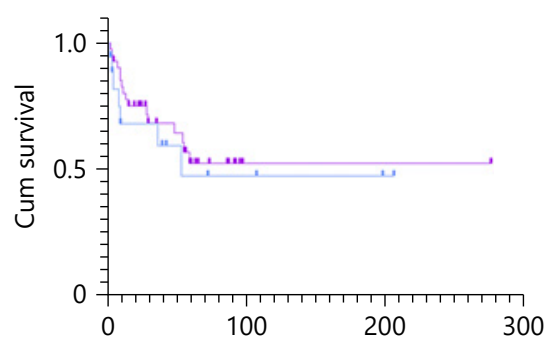

a2 Recurrence-free survival time, months

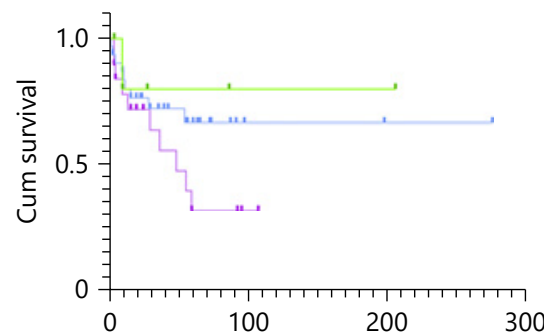

b2 Recurrence-free survival time, months

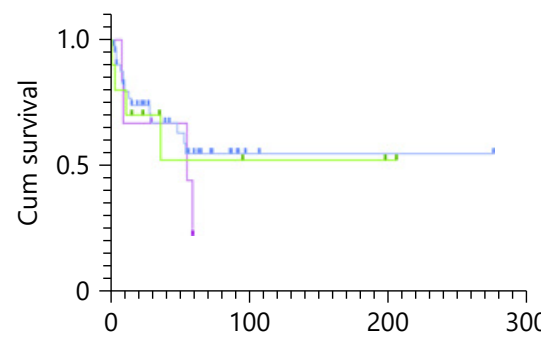

c2 Recurrence-free survival time, months

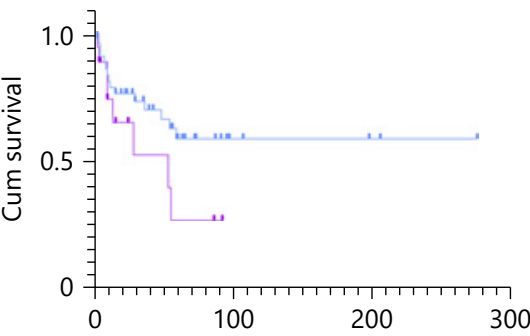

d2 Recurrence-free survival time, months



e2 Recurrence-free survival time, months 5 
reported as a safe, feasible, minimally invasive approach for UrCs [19]. Bilateral lymphadenectomy made sense in helping guide the evaluation of staging and prognosis, and it may be therapeutic for at least some patients [18, $20]$. In the current series, $74.21 \%$ of UrC patients underwent extended partial cystectomy, $36.21 \%$ of these cases were performed bilateral pelvic lymph nodes dissection, and $36.36 \%$ were positive lymph node. A striking finding in our study was the local progression (local abdominal metastasis or lymph node metastasis) or existence of organ metastasis at diagnosis significantly determined shorter CSS compared with the patients of nonprogression (T1-3N0M0). Therefore, early diagnosis and surgical intervention are essential to prolonging survival.

Previously, UrC was usually recognized as a malignant tumor with a high degree of malignancy accompanied by a poor prognosis. According to the records of SEER and RKI databases, the relative 5-year CSS rate was $54.8 \%$ in Germany and $64.4 \%$ in the USA [21]. Our results showed that the 3 -year CSS rate was $69.1 \%$ and the 5 -year CSS rate was $61.2 \%$, which was approximately similar to Western populations. Although early interventions for patients with $\mathrm{UrC}$ are necessary, the risk factors affecting the prognosis were still unclear. Several parameters had been discussed such as age, sex, Sheldon stage, Mayo stage, tumor grade, LN status, presence of distant metastasis, positive surgical margin, tumor size, pathologic type, and surgical intervention. Szarvas et al. [14] meta-analysis revealed Sheldon stage 4IIIB, Mayo stage 4II, presence of LN or distant metastases, positive surgical margin, and ECOG performance status were independent risk predictors after multivariable analyses. Other previous evidence showed that the histologic tumor stage and negative surgical margins were the most significant predictors of survival for $\operatorname{UrC}[5,11,18]$. In our study, LN or abdominal local progression or distant metastases were validated to have a significant correlation with OS and CSS. While the OS and CSS of these patients with urachal cancer were not associated with the pathological tumor stage, tumor grade, and surgical procedure, additionally, a positive surgical margin affected much lower CSS rate and RFS rate significantly. Therefore, more prospective, controlled studies on the surgical approach and extent of resection are to be expected and conducted.

Since UrC has a high risk of recurrence and distant metastases and it is extremely poor for the prognosis of patients with recurrent and/or metastatic UrC. The point that treatment is different for localized or metastatic disease has been agreed with by many researchers [6]. However, there is still no standard chemotherapy regimen or radiation strategy for advanced or metastatic UrC. Due to the histological similarity to colorectal adenocarcinoma and urothelial carcinomas, cisplatin and 5-FU are the most common chemotherapeutic agents used for UrC $[14,17,22]$. Yanagihara et al. [23] shared with us 2 cases of successful treatment of UrC with FOLFOX6 regimen. Kawakami et al. [24] reported 2 patients with recurrent and metastatic UrC had survived with a RFS period of more than 10 years after treated by combinations of surgery, radiation, and chemotherapy. Recently, a metaanalysis indicated that 5-FU-based chemotherapy was more effective than cisplatin-based regimens in the treatment of UrC, and the combinations of 5-FU with cisplatin could provide the strongest antitumor effect [14]. In our series, one case, performed extended partial cystectomy followed by adjuvant chemotherapy-5-FU-based regimen, had survived for more than 15 years without recurrence or metastasis. These experiences suggested that multimodality therapy may be beneficial for some patients with UrC.

The molecular characterization of $\mathrm{UrC}$ remained one of the topics of current research. Notably, the spectrum of molecular alterations in urachal tumors was more similar to primary urinary bladder adenocarcinoma and colorectal adenocarcinoma compared with conventional urothelial carcinoma [25]. Recurrent KRAS, NRAS, BRAF, APC, TP53, NF1, PTEN, and/or SMAD4 mutations were usually detected, meanwhile, TERT promoter and PIK3CA mutations, which were common in conventional urothelial carcinoma, were proved rare in $\mathrm{UrC}[22$, 26-29]. This also explained the prognostic difference between the UrC and BC. A recent study showed detectable genetic alterations in PD-L1 status, intracellular signaltransduction pathways (RAS/RAF/PI3K), ERBB2 (HER2), MET, FGFR1, and PDGFRA [30]. These findings might provide new evidence and ideas for future immunotherapy and targeted therapeutic strategies for UrC.

\section{Conclusions}

In conclusion, our study showed that $\mathrm{UrC}$ was a rare, high-risk neoplasm. The majority of UrC were adenocarcinomas. The results indicated that high-risk TNM stage (with LN or abdominal local progression or distant metastases) and positive surgical margin were the most important predictor of prognosis. Localized T stage, histologic grade, and surgical procedure cause no significant effect on patient prognosis. Extended partial cystectomy with en bloc resection of the entire urachus was the recommended 
surgical approach of patients with UrC. Active multimodal treatments combined with surgery may improve the survival of patients with recurrent and metastatic disease.

\section{Acknowledgments}

The authors are grateful to all the colleagues who helped in the preparation of this article.

\section{Statement of Ethics}

The protocol of our study followed the World Medical Association Declaration of Helsinki. The trial was approved by the local Ethics Committee of Qilu Hospital, Shandong University. Approval reference No: KYLL-2021(KS)-1008. All enrolled patients were informed of the trial and signed informed consents.

\section{Conflict of Interest Statement}

The authors declare that they have no competing interests.

\section{Author Contributions}

G.J. Shao and C.R. Xu contributed equally to this article. G.J. Shao was the first author and C.R. Xu was the co-first author. Y.D. Fan and L.Q. Zhou were the equally correspondent author of this article. All authors are accountable for all aspects of the work. All authors approved the final article. G.J. Shao and Y.D. Fan involved in conceptualization. G.J. Shao and C.R. Xu involved in formal analysis. G.J. Shao and X.Q. Zhang involved in investigation. G.J. Shao, C.R. Xu, J.K. Liu, and X.S. Li involved in methodology. Y.D. Fan, X.S. Li, and L.Q. Zhou. involved in project administration. G.J. Shao, X.Q. Zhang, J.K. Liu, and L.C. Li involved in resource acquiring. Y.D. Fan, X.S. Li, and L.Q. Zhou involved in supervision. C.R. Xu and X.F. Li involved in visualization. G.J. Shao and C.R. Xu involved in writing - original draft. G.J. Shao, C.R. Xu, Y.D. Fan, and L.Q. Zhou involved in writing - review and editing.

\section{Data Availability Statement}

All data generated or analyzed during this study were included in this article. Further enquiries can be directed to the corresponding author.

\section{Funding Sources}

No funding was received for this article.

\section{References}

1 Frimberger DC, Kropp BP. Bladder anomalies in children. Campbell-Walsh Urol. 2012:3379-88.

2 Gopalan A, Sharp DS, Fine SW, Tickoo SK, Herr HW, Reuter VE, et al. Urachal carcinoma: a clinicopathologic analysis of 24 cases with outcome correlation. Am J Surg Pathol. 2009 May;33(5):659-68.

3 Paras FA, MacLennan GT. Urachal adenocarcinoma. J Urol. 2008;180(2):720-0.

4 Wright JL, Porter MP, Li CI, Lange PH, Lin DW. Differences in survival among patients with urachal and nonurachal adenocarcinomas of the bladder. Cancer. 2006 Aug 15; 107(4):721-8.

5 Dhillon J, Liang Y, Kamat AM, SiefkerRadtke A, Dinney CP, Czerniak B, et al. Urachal carcinoma: a pathologic and clinical study of 46 cases. Hum Pathol. 2015 Dec; 46(12):1808-14.

6 Claps M, Stellato M, Zattarin E, Mennitto A, Sepe P, Guadalupi V, et al. Current understanding of urachal adenocarcinoma and management strategy. Curr Oncol Rep. 2020;22(1):9.

7 Molina JR, Quevedo JF, Furth AF, Richardson RL, Zincke H, Burch PA. Predictors of survival from urachal cancer: a Mayo Clinic study of 49 cases. Cancer. 2007 Dec 1; 110(11):2434-40.
8 Begg RC. The Urachus: its anatomy, histology and development. J Anat. 1930 Jan; 64(Pt 2):170-83.

9 Riva G, Mian C, Luchini C, Girolami I, Ghimenton C, Cima L, et al. Urachal carcinoma: from gross specimen to morphologic, immunohistochemical, and molecular analysis. Virchows Arch. 2019 Jan;474(1):13-20.

10 Paner GP, Barkan GA, Mehta V, Sirintrapun SJ, Tsuzuki T, Sebo TJ, et al. Urachal carcinomas of the nonglandular type: salient features and considerations in pathologic diagnosis. Am J Surg Pathol. 2012 Mar;36(3): 432-42.

11 Ashley RA, Inman BA, Sebo TJ, Leibovich BC, Blute ML, Kwon ED, et al. Urachal carcinoma: clinicopathologic features and long-term outcomes of an aggressive malignancy. Cancer. 2006 Aug 15;107(4):712-20.

12 Sheldon CA, Clayman RV, Gonzalez R, Williams RD, Fraley EE. Malignant urachal lesions. J Urol. 1984 Jan;131(1):1-8.

13 Pinthus JH, Haddad R, Trachtenberg J, Holowaty E, Bowler J, Herzenberg AM, et al. Population based survival data on urachal tumors. J Urol. 2006 Jun;175(6):2042-7; discussion 47.
14 Szarvas T, Módos O, Niedworok C, Reis H, Szendröi A, Szász MA, et al. Clinical, prognostic, and therapeutic aspects of urachal carcinoma: a comprehensive review with meta-analysis of 1,010 cases. Urol Oncol. 2016 Sep;34(9):388-98.

15 Thali-Schwab CM, Woodward PJ, Wagner BJ. Computed tomographic appearance of urachal adenocarcinomas: review of 25 cases. Eur Radiol. 2005 Jan;15(1):79-84.

16 Aggarwal A, Agarwal S, Pandey S, Sankhwar S. Urachal adenocarcinoma. BMJ Case Rep. 2018 Sep;2018:152018.

17 Siefker-Radtke AO, Gee J, Shen Y, Wen S, Daliani D, Millikan RE, et al. Multimodality management of urachal carcinoma: the $\mathrm{M}$. D. Anderson Cancer Center experience. J Urol. 2003 Apr;169(4):1295-8.

18 Herr HW, Bochner BH, Sharp D, Dalbagni G, Reuter VE. Urachal carcinoma: contemporary surgical outcomes. J Urol. $2007 \mathrm{Jul}$; 178(1):74-8; discussion 78.

19 Wadhwa P, Kolla SB, Hemal AK. Laparoscopic en bloc partial cystectomy with bilateral pelvic lymphadenectomy for urachal adenocarcinoma. Urology. 2006 Apr;67(4):837-43.

20 Duan F, Zhai W, Zhang B, Guo S. Urachal carcinoma: impact of recurrence pattern and lymphadenectomy on long-term outcomes. Cancer Med. 2020 Jun;9(12):4166-74. 
21 Hager T, Kraywinkel K, Szarvas T, Hadaschik B, Schmid KW, Reis H. Urachal cancer in Germany and the USA: an RKI/SEER population-based comparison study. Urol Int. 2020;104(9-10):803-9.

22 Módos $\mathrm{O}$, Reis $\mathrm{H}$, Niedworok C, Rübben $\mathrm{H}$, Szendröi A, Szász MA, et al. Mutations of KRAS, NRAS, BRAF, EGFR, and PIK3CA genes in urachal carcinoma: occurrence and prognostic significance. Oncotarget. 2016 Jun 28;7(26):39293-301.

23 Yanagihara Y, Tanji N, Miura N, Shirato A, Nishimura K, Fukumoto T, et al. Modified FOLFOX6 chemotherapy in patients with metastatic urachal cancer. Chemotherapy. 2013;59(6):402-6.
24 Kawakami S, Kageyama Y, Yonese J, Fukui I, Kitahara S, Arai G, et al. Successful treatment of metastatic adenocarcinoma of the urachus: report of 2 cases with more than 10year survival. Urology. 2001 Sep;58(3):462.

25 Taylor AS, Mehra R, Udager AM. Glandular tumors of the urachus and urinary bladder: a practical overview of a broad differential diagnosis. Arch Pathol Lab Med. 2018 Oct; 142(10):1164-76.

26 Collazo-Lorduy A, Castillo-Martin M, Wang L, Patel V, Iyer G, Jordan E, et al. Urachal carcinoma shares genomic alterations with colorectal carcinoma and may respond to epidermal growth factor inhibition. Eur Urol. 2016 Nov;70(5):771-5.
27 Lee S, Lee J, Sim SH, Lee Y, Moon KC, Lee $\mathrm{C}$, et al. Comprehensive somatic genome alterations of urachal carcinoma. J Med Genet. 2017 Aug;54(8):572-8.

28 Thiem S, Herold T, Krafft U, Bremmer F, Tolkach Y, Szász AM, et al. Telomerase reverse transcriptase (TERT) promoter mutations are rare in urachal cancer. Pathol Int. 2017 Dec;67(12):597-601.

29 Nagy N, Reis H, Hadaschik B, Niedworok C, Módos O, Szendrői A, et al. Prevalence of APC and PTEN alterations in urachal cancer. Pathology oncology research. Pathol Oncol Res. 2020 Oct;26(4):2773-81.

30 Reis H, van der Vos KE, Niedworok C, Herold T, Módos O, Szendrői A, et al. Pathogenic and targetable genetic alterations in 70 urachal adenocarcinomas. Int $J$ Cancer. 2018 Oct 1;143(7):1764-73. 